\title{
Set-Theoretic Model Reference Adaptive Control for Performance Guarantees in Human-in-the-Loop Systems; A Pilot Study
}

\author{
Ahmet Taha Koru* \\ Pennsylvania State University, State College, PA, 16803, USA \\ K Merve Dogan, Tansel Yucelen \\ University of South Florida, Tampa, FL, 33620, USA \\ Ehsan Arabi \\ University of Michigan, Ann Arbor, MI, 48109, USA \\ Rifat Sipahi \\ Northeastern University, Boston, MA, 02115, USA \\ Yildiray Yildiz \\ Bilkent University, Ankara, Turkey
}

\begin{abstract}
Control design that achieves high performance in human-in-the-loop machine systems still remains a challenge. Model reference adaptive control (MRAC) is well positioned for this need since it can help address issues of nonlinearities and uncertainties in the machine system. Moreover, given that human behavior is also nonlinear, task-dependent, and timevarying in nature, MRAC could also offer solutions for a highly synergistic human-machine interactions. Recent results on set-theoretic MRAC further our understanding in terms of designing controllers that can bring the behavior of nonlinear machine dynamics within a tolerance of the behavior of a reference model; that is, such controllers can make nonlinear and uncertain dynamics behave like a "nominal model." The advantage of this argument is that humans can be trained only with nominal models, without overwhelming them with extensive training on complex, nonlinear dynamics. Even only with a training on simple nominal models, human commands when supplemented with set-theoretic MRAC can help control complex, nonlinear dynamics. In this study, we present a computer-based simulator that our research team tested under various conditions, as preliminary results supporting the promise of a simpler yet more effective means to train humans and to still achieve satisfactory performance in human-machine systems where humans are presented with complex, nonlinear dynamics.
\end{abstract}

\section{Introduction}

Many safety-critical systems interact with a human being, i.e., fly-by-wire aircraft control systems (interacting with a pilot), automobiles with driver assistance systems (interacting with a driver), and medical devices (interacting with a doctor, nurse, or patient) [1]. The human supervision and control skills play a central role in such systems, since with the human operator the feedback loop is closed and human presence could lead to poor performance or instability [2]. This is clearly exemplified, for example, in pilot-airplane and driver-automobile interactions [3-9].

One of the challenges in analysis and design of human-in-the-loop systems is deriving models of human behaviors. The most of the mathematical models proposed in the literature are linear time-invariant, see, e.g., [5, 8, 9]. However, human behavior is much more complex due to physiological, psychological and behavioral aspects of human beings [10]. In fact, human behavior is time-varying, task-dependent and involve considerable nonlinearities [11, 12]. Therefore, the control design of human-in-the-loop systems to guarantee some level of closed-loop performance is challenging, due to nonlinearities, time-varying effects, and/or uncertainties.

\footnotetext{
*Author of Correspondence. Postdoctoral Scholar, the Department of Aerospace Engineering, e-mail: ahtakoru@gmail.com
} 
Considering the discussion above, MRAC has a strategic importance, since this control approach is a powerful tool to address the issues of nonlinearities and uncertainties, see for example [13]. Furthermore, recent results on set-theoretic MRAC further our understanding in terms of designing controllers that can bring the behavior of nonlinear machine dynamics within a tolerance of the behavior of a reference model; that is, such controllers can make nonlinear and uncertain dynamics behave like a "nominal model" [14]. In [15], it is shown that guaranteeing a user-defined performance constraint on the norm of system trajectory error ultimately results in an acceptable human performance in accomplishing a given task. Within the context of the cited study, the results are theoretically proven assuming LTI models for humans. We then wonder whether or not qualitatively and/or quantitatively similar arguments could be made a human-machine system where real humans are involved.

To this end, we designed a computer-based cursor-tracking simulation game where humans interact with an uncertain dynamical system via a mouse interface. The physical system is visualized to provide feedback to the human where the human is tasked to drive the physical system shown from its initial state to a reference terminal state. After testing the game with the nominal system, our research team then tested the game in the presence of uncertainties. These test results are presented here as a pilot, preliminary study, which indeed support the idea that human commands when supplemented with set-theoretic MRAC can help control complex, nonlinear dynamics; even when trained only with a nominal model.

\section{Mathematical Preliminaries}

\section{A. Notation and Necessary Definitions}

In this paper, we write $\mathbb{R}$ to denote the set of real numbers, $\mathbb{C}$ to denote the set of complex numbers, $\mathbb{R}^{n}$ to denote the set of $n \times 1$ real column vectors, $\mathbb{R}^{n \times m}$ to denote the set of $n \times m$ real matrices, $\mathbb{R}_{+}$to denote the set of positive real numbers, $\mathbb{R}_{+}^{n \times n}$ to denote the set of $n \times n$ positive definite matrices, $\mathbb{D}^{n \times n}$ to denote the set of $n \times n$ real matrices with diagonal scalar entries, $0_{n \times n}$ to denote the $n \times n$ zero matrix, and " $\triangleq$ " to denote equality by definition. In addition, $(\cdot)^{\mathrm{T}}$ denotes the transpose, $(\cdot)^{-1}$ denotes the inverse, $\operatorname{tr}(\cdot)$ denotes the trace, $\|\cdot\|_{2}$ denotes the Euclidean norm, $\|\cdot\|_{\mathrm{F}}$ denotes the Frobenius norm, and $\|A\|_{2} \triangleq \sqrt{\lambda_{\max }\left(A^{\mathrm{T}} A\right)}$ denotes the induced 2-norm of the matrix $A \in \mathbb{R}^{n \times m}$.

The following definitions are necessary for the presented results in this paper.

Definition 1 (Projection Operator, [16, 17]) Let $\Omega=\left\{\theta \in \mathbb{R}^{n}:\left(\theta_{i}^{\min } \leq \theta_{i} \leq \theta_{i}^{\max }\right)_{i=1,2, \cdots, n}\right\}$ be a convex hypercube in $\mathbb{R}^{n}$, where $\left(\theta_{i}^{\min }, \theta_{i}^{\max }\right)$ represent the minimum and maximum bounds for the $i^{\text {th }}$ component of the $n$-dimensional parameter vector $\theta$. In addition, for a sufficiently small positive constant $v$, a second hypercube is defined by $\Omega_{v}=\left\{\theta \in \mathbb{R}^{n}:\left(\theta_{i}^{\min }+v \leq \theta_{i} \leq \theta_{i}^{\max }-v\right)_{i=1,2, \cdots, n}\right\}$, where $\Omega_{v} \subset \Omega$. The projection operator Proj $: \mathbb{R}^{n} \times \mathbb{R}^{n} \rightarrow \mathbb{R}^{n}$ is then defined component-wise by $\operatorname{Proj}(\theta, y) \triangleq\left(\frac{\theta_{i}^{\max }-\theta_{i}}{v}\right) y_{i}$, if $\theta_{i}>\theta_{i}^{\max }-v$ and $y_{i}>0, \operatorname{Proj}(\theta, y) \triangleq\left(\frac{\theta_{i}-\theta_{i}^{\min }}{v}\right) y_{i}$, if $\theta_{i}<\theta_{i}^{\min }+v$ and $y_{i}<0$, and $\operatorname{Proj}(\theta, y) \triangleq y_{i}$, otherwise, where $y \in \mathbb{R}^{n}$ [16]. Based on this definition and $\theta^{*} \in \Omega_{\gamma}$, note that

$$
\left(\theta-\theta^{*}\right)^{\mathrm{T}}(\operatorname{Proj}(\theta, y)-y) \leq 0,
$$

holds for $\theta \in \Omega$ and $y \in \mathbb{R}^{n}\left[16\right.$ [17]. This definition can be further generalized to matrices as $\operatorname{Proj}_{\mathrm{m}}(\Theta, Y)=$

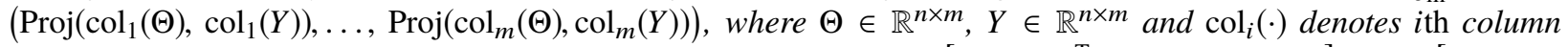
operator. In this case, for a given matrix $\Theta^{*}$ it follows from $(1)$ that $\operatorname{tr}\left[\left(\Theta-\Theta^{*}\right)^{\mathrm{T}}\left(\operatorname{Proj}_{\mathrm{m}}(\Theta, Y)-Y\right)\right]=\sum_{i=1}^{m}\left[\operatorname{col}_{i}(\Theta-\right.$

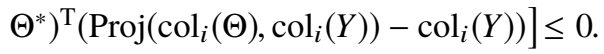

Definition 2 (Generalized Restricted Potential Function, [18]) Let $\|z\|_{H}=\sqrt{z^{\mathrm{T}} H z}$ be a weighted Euclidean norm, where $z \in \mathbb{R}^{p}$ is a real column vector and $H \in \mathbb{R}_{+}^{p \times p}$. We define $\phi\left(\|z\|_{\mathrm{H}}\right), \phi: \mathbb{R} \rightarrow \mathbb{R}$, to be a generalized restricted potential function (generalized barrier Lyapunov function) on the set

$$
\mathcal{D}_{\epsilon} \triangleq\left\{z:\|z\|_{\mathrm{H}} \in[0, \epsilon)\right\}
$$

with $\epsilon \in \mathbb{R}_{+}$being a-priori, user-defined constant, if the following statements hold [18]: i) If $\|z\|_{\mathrm{H}}=0$, then $\phi\left(\|z\|_{\mathrm{H}}\right)=0$. ii) If $z \in \mathcal{D}_{\epsilon}$ and $\|z\|_{\mathrm{H}} \neq 0$, then $\phi\left(\|z\|_{\mathrm{H}}\right)>0$. iii) If $\|z\|_{\mathrm{H}} \rightarrow \epsilon$, then $\phi\left(\|z\|_{\mathrm{H}}\right) \rightarrow \infty$. iv) $\phi\left(\|z\|_{\mathrm{H}}\right)$ is continuously differentiable on $\mathcal{D}_{\epsilon}$. v) If $z \in \mathcal{D}_{\epsilon}$, then $\phi_{d}\left(\|z\|_{\mathrm{H}}\right)>0$, where $\phi_{d}\left(\|z\|_{\mathrm{H}}\right) \triangleq \mathrm{d} \phi\left(\|z\|_{\mathrm{H}}\right) / \mathrm{d}\|z\|_{\mathrm{H}}^{2}$. vi) If $z \in \mathcal{D}_{\epsilon}$, then $2 \phi_{d}\left(\|z\|_{\mathrm{H}}\right)\|z\|_{\mathrm{H}}^{2}-\phi\left(\|z\|_{\mathrm{H}}\right)>0$. 


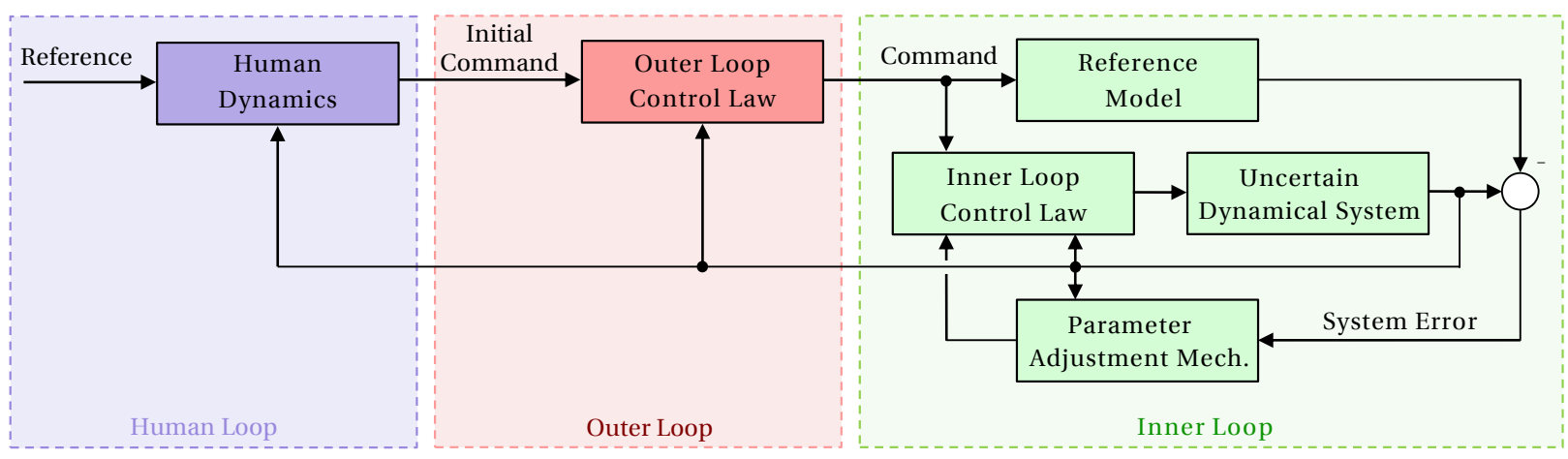

Fig. 1 Block diagram of the human-in-the-loop model reference adaptive control architecture [15].

\section{B. Problem Formulation}

In this paper, we consider human-in-the-loop physical systems with inner and outer feedback control loops as also considered in [15]. The control objective is to have the uncertain machine at the inner loop to behave close to an ideal system that corresponds to the case where there is no uncertainties present in the system. To this end, the machine behavior $y(t)$ is observed by the human and an initial command is generated based on a reference command. The initial command passes through an outer loop to generate the actual command for the machine. The outer loop dynamics can serve as an intermediate step for employing either sequential loop closure and/or high-level guidance methods [19, 20] (see Figure 11). Note that, the system structure considered in [21] can be viewed as a special case of this architecture when the inner and outer loops are combined.

In what follows, we present the system dynamics corresponding to each of the feedback loops. We start with the inner loop where we consider the uncertain dynamics representing a physical system given by

$$
\dot{x}^{*}(t)=\left[\begin{array}{cc}
A_{\mathrm{p}} & 0_{n_{\mathrm{p}} \times n_{\phi_{\mathrm{p}}}} \\
G_{\mathrm{p}} & F_{\mathrm{p}}
\end{array}\right] x^{*}(t)+\left[\begin{array}{c}
B_{\mathrm{p}} \\
0_{n_{\mathrm{c}} \times m}^{\mathrm{T}}
\end{array}\right]\left(\Lambda u(t)+\delta_{\mathrm{p}}\left(x_{\mathrm{p}}(t)\right)\right),
$$

where $A_{\mathrm{p}} \in \mathbb{R}^{n_{\mathrm{p}} \times n_{\mathrm{p}}}, B_{\mathrm{p}} \in \mathbb{R}^{n_{\mathrm{p}} \times m}, F_{\mathrm{p}} \in \mathbb{R}^{n_{\phi_{\mathrm{p}}} \times n_{\phi_{\mathrm{p}}}}$, and $G_{\mathrm{p}} \in \mathbb{R}^{n_{\phi_{\mathrm{p}}} \times n_{\mathrm{p}}}$ are the system matrices, $u(t) \in \mathbb{R}^{m}$ is the control input, $\delta_{\mathrm{p}}: \mathbb{R}^{n_{\mathrm{p}}} \rightarrow \mathbb{R}^{m}$ is a system uncertainty, $\Lambda \in \mathbb{R}_{+}^{m \times m} \cap \mathbb{D}^{m \times m}$ is an unknown control effectiveness matrix, and we assume that the overall system is controllable. In (3) $x^{*}(t)$ is the system state containing the primary measurable

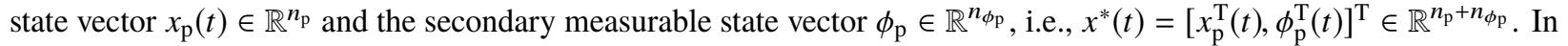
addition, we assume that the system uncertainty $\delta_{\mathrm{p}}\left(x_{\mathrm{p}}(t)\right)$ is parameterized as

$$
\delta_{\mathrm{p}}\left(x_{\mathrm{p}}(t)\right)=W_{\mathrm{p}}^{\mathrm{T}}(t) \sigma_{\mathrm{p}}\left(x_{\mathrm{p}}(t)\right),
$$

where $W_{\mathrm{p}}(t) \in \mathbb{R}^{s \times m}$ is a bounded unknown weight matrix (i.e., $\left\|W_{0}(t)\right\|_{\mathrm{F}} \leq w_{0}$ ) with a bounded time rate of change (i.e., $\left.\left\|\dot{W}_{0}(t)\right\|_{\mathrm{F}} \leq \dot{w}_{0}\right)$ and $\sigma_{\mathrm{p}}: \mathbb{R}^{n_{\mathrm{p}}} \rightarrow \mathbb{R}^{s}$ is a known basis function of the form

$$
\sigma_{\mathrm{p}}\left(x_{\mathrm{p}}(t)\right)=\left[\sigma_{\mathrm{p} 1}\left(x_{\mathrm{p}}(t)\right), \sigma_{\mathrm{p} 2}\left(x_{\mathrm{p}}(t)\right), \ldots, \sigma_{\mathrm{p} s}\left(x_{\mathrm{p}}(t)\right)\right]^{\mathrm{T}} .
$$

Hence, one can equivalently write $(3)$ as

$$
\begin{aligned}
& \dot{x}_{\mathrm{p}}(t)=A_{\mathrm{p}} x_{\mathrm{p}}(t)+B_{\mathrm{p}} \Lambda u(t)+B_{\mathrm{p}} W_{\mathrm{p}}^{\mathrm{T}}(t) \sigma_{\mathrm{p}}\left(x_{\mathrm{p}}(t)\right), \quad x_{\mathrm{p}}(0)=x_{\mathrm{p} 0}, \\
& \dot{\phi}_{\mathrm{p}}(t)=F_{\mathrm{p}} \phi_{\mathrm{p}}(t)+G_{\mathrm{p}} x_{\mathrm{p}}(t), \quad \phi_{\mathrm{p}}(0)=\phi_{\mathrm{p} 0} .
\end{aligned}
$$

Note that (5) is used at the inner loop for the control design and (6) is used at the outer loop for generating the command signal that is fed to the inner loop.

To address command following, we consider the dynamic compensator state $x_{\mathrm{c}}(t) \in \mathbb{R}^{n_{\mathrm{c}}}$ satisfying

$$
\begin{aligned}
& \dot{x}_{\mathrm{c}}(t)=A_{\mathrm{c}} x_{\mathrm{c}}(t)+B_{\mathrm{c}} e_{\mathrm{p}}(t), \quad x_{\mathrm{c}}(0)=x_{\mathrm{c} 0}, \\
& z_{\mathrm{c}}(t)=C_{\mathrm{c}} x_{\mathrm{c}}(t)+D_{\mathrm{c}} e_{\mathrm{p}}(t),
\end{aligned}
$$


where $A_{\mathrm{c}} \in \mathbb{R}^{n_{\mathrm{c}} \times n_{\mathrm{p}}}, B_{\mathrm{c}} \in \mathbb{R}^{n_{\mathrm{c}} \times n_{y}}, C_{\mathrm{c}} \in \mathbb{R}^{n_{\mathrm{z}} \times n_{\mathrm{c}}}, D_{\mathrm{c}} \in \mathbb{R}^{n_{\mathrm{z}} \times n_{y}}, z(t) \in \mathbb{R}^{z}$ is the output of the dynamic compensator, $e_{\mathrm{p}}(t) \triangleq y(t)-c(t)$, and $y(t) \triangleq C_{\mathrm{p}} x_{\mathrm{p}}(t)$ with $C_{\mathrm{p}} \in \mathbb{R}^{n_{y} \times n_{\mathrm{p}}}$. We now consider the inner loop control law given by

$$
u(t)=u_{\mathrm{n}}(t)+u_{\mathrm{a}}(t)
$$

where $u_{\mathrm{n}}(t) \in \mathbb{R}^{m}$ and $u_{\mathrm{a}}(t) \in \mathbb{R}^{m}$ are the nominal and adaptive control laws, respectively. Furthermore, let the nominal control law be

$$
u_{\mathrm{n}}(t)=-K_{\mathrm{p}} x_{\mathrm{p}}(t)-K_{\mathrm{c}} z_{\mathrm{c}}(t),
$$

with $K_{\mathrm{p}} \in \mathbb{R}^{m \times n_{\mathrm{p}}}$ and $K_{\mathrm{c}} \in \mathbb{R}^{m \times n_{z}}$. Now, (5) can be augmented with (7) as

$$
\dot{x}(t)=A_{\mathrm{r}} x(t)+B_{\mathrm{r}} c(t)+B \Lambda\left(u_{\mathrm{a}}(t)+W^{\mathrm{T}}(t) \sigma(x(t), c(t))\right), \quad x(0)=x_{00},
$$

where $x(t) \triangleq\left[x_{\mathrm{p}}^{\mathrm{T}}(t), x_{\mathrm{c}}^{\mathrm{T}}(t)\right]^{\mathrm{T}} \in \mathbb{R}^{n}, n=n_{\mathrm{p}}+n_{\mathrm{c}}$, is the augmented state vector, $W(t) \triangleq\left[W_{\mathrm{p}}^{\mathrm{T}}(t),\left(\Lambda^{-1}-I_{m \times m}\right)\left(K_{\mathrm{p}}+\right.\right.$ $\left.\left.K_{\mathrm{c}} D_{\mathrm{c}} C_{\mathrm{p}}\right),\left(\Lambda^{-1}-I_{m \times m}\right) K_{\mathrm{c}} C_{\mathrm{c}},-\left(\Lambda^{-1}-I_{m \times m}\right) K_{\mathrm{c}} D_{\mathrm{c}}\right]^{\mathrm{T}} \in \mathbb{R}^{\left(s+n+n_{y}\right) \times m}$ is an unknown (aggregated) weight matrix, $\sigma(x(t), c(t)) \triangleq\left[\sigma_{\mathrm{p}}^{\mathrm{T}}\left(x_{\mathrm{p}}(t)\right), x_{\mathrm{p}}^{\mathrm{T}}(t), x_{\mathrm{c}}^{\mathrm{T}}(t), c(t)\right]^{\mathrm{T}} \in \mathbb{R}^{s+n+n_{y}}$ is a known (aggregated) basis function, $x_{00} \triangleq\left[x_{\mathrm{p} 0}^{\mathrm{T}}, x_{\mathrm{c} 0}^{\mathrm{T}}\right]^{\mathrm{T}}$,

$$
\begin{aligned}
A_{\mathrm{r}} & \triangleq\left[\begin{array}{cc}
A_{\mathrm{p}}-B_{\mathrm{p}} K_{\mathrm{p}}-B_{\mathrm{p}} K_{\mathrm{c}} D_{\mathrm{c}} C_{\mathrm{p}} & -B_{\mathrm{p}} K_{\mathrm{c}} C_{\mathrm{c}} \\
B_{\mathrm{c}} C_{\mathrm{p}} & A_{\mathrm{c}}
\end{array}\right] \in \mathbb{R}^{n \times n}, \\
B_{\mathrm{r}} & \triangleq\left[\begin{array}{c}
B_{\mathrm{p}} K_{\mathrm{c}} D_{\mathrm{c}} \\
-B_{\mathrm{c}}
\end{array}\right] \in \mathbb{R}^{n \times n_{y}}, \quad B \triangleq\left[\begin{array}{ll}
B_{\mathrm{p}}^{\mathrm{T}} & 0_{n_{\mathrm{c}} \times m}^{\mathrm{T}}
\end{array}\right]^{\mathrm{T}} \in \mathbb{R}^{n \times m} .
\end{aligned}
$$

We now consider the adaptive control law given by

$$
u_{\mathrm{a}}(t)=-\hat{W}^{\mathrm{T}}(t) \sigma(x(t), c(t)) .
$$

In [14], $\hat{W}(t) \in \mathbb{R}^{\left(s+n+n_{y}\right) \times m}$ is the estimate of $W(t)$ satisfying the update law given by

$$
\dot{\hat{W}}(t)=\gamma \operatorname{Proj}_{\mathrm{m}}\left(\hat{W}(t), \phi_{d}\left(\|e(t)\|_{P}\right) \sigma(x(t)) e^{\mathrm{T}}(t) P B\right), \quad \hat{W}(0)=\hat{W}_{0},
$$

with $\hat{W}_{\max }$ being the projection norm bound, $\gamma \in \mathbb{R}_{+}$being the learning rate (i.e., adaptation gain), and $P \in \mathbb{R}_{+}^{n \times n}$ being a solution of the Lyapunov equation given by

$$
0=A_{\mathrm{r}}^{\mathrm{T}} P+P A_{\mathrm{r}}+R,
$$

where $R \in \mathbb{R}_{+}^{n \times n}$. In addition, $e(t) \triangleq x(t)-x_{\mathrm{r}}(t)$ in $(15)$ is the system error with $x_{\mathrm{r}}(t) \in \mathbb{R}^{n}$ being the reference state vector of a reference model dynamics at the inner loop that captures a desired inner loop dynamical system performance given by

$$
\dot{x}_{\mathrm{r}}(t)=A_{\mathrm{r}} x_{\mathrm{r}}(t)+B_{\mathrm{r}} c(t), \quad x_{\mathrm{r}}(0)=x_{\mathrm{r} 0} .
$$

Using [14, 15, and (17), the inner loop system error dynamics is given by

$$
\begin{aligned}
\dot{e}(t) & =A_{\mathrm{r}} e(t)-B \Lambda \tilde{W}^{\mathrm{T}}(t) \sigma(x(t), c(t)), \quad e(0)=e_{0}, \\
\dot{\tilde{W}}(t) & =\gamma \operatorname{Proj}_{\mathrm{m}}\left(\hat{W}(t), \phi_{d}\left(\|e(t)\|_{P}\right) \sigma(x(t), c(t)) e^{\mathrm{T}}(t) P B\right)-\dot{W}(t), \quad \tilde{W}(0)=\tilde{W}_{0},
\end{aligned}
$$

where $\tilde{W}(t) \triangleq \hat{W}(t)-W(t) \in \mathbb{R}^{\left(s+n+n_{y}\right) \times m}$ is the weight estimation error and $e_{0} \triangleq x_{00}-x_{\mathrm{r} 0}$. Once again, we note that the unknown weight matrix $W(t)$ and its derivative have unknown upper bounds (i.e., $\|W(t)\|_{\mathrm{F}} \leq w$ and $\|\dot{W}(t)\|_{\mathrm{F}} \leq \dot{W}$ with unknown $w$ and $\dot{w}$ ).

For the outer loop, we consider the dynamic compensator based on (6) as

$$
\begin{aligned}
\dot{\phi}_{\mathrm{c}}(t) & =F_{\mathrm{c}} \phi_{\mathrm{c}}(t)+G_{\mathrm{c}} \eta_{\mathrm{p}}(t), \quad \phi_{\mathrm{p}}(0)=\phi_{\mathrm{p} 0}, \\
c(t) & =H_{\mathrm{c}} \phi_{\mathrm{c}}(t)-J_{\mathrm{c}} \eta_{\mathrm{p}}(t), \\
\eta_{\mathrm{p}}(t) & =M_{\mathrm{p}} \phi_{\mathrm{p}}(t)-c_{0}(t),
\end{aligned}
$$




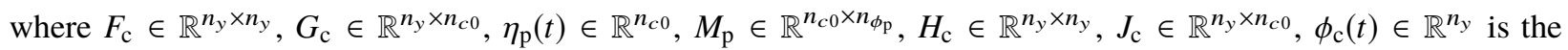
outer loop state vector, $c_{0}(t) \in \mathbb{R}^{n_{c 0}}$ is the initial command signal produced by the human, which is the input to the outer loop architecture, and $c(t) \in \mathbb{R}^{n_{y}}$ is the generated command at the outer loop as shown in Figure 1 . Letting $\phi(t)=\left[x_{\mathrm{r}}^{\mathrm{T}}(t), \phi_{\mathrm{p}}^{\mathrm{T}}(t), \phi_{\mathrm{c}}^{\mathrm{T}}(t)\right]^{\mathrm{T}} \in \mathbb{R}^{n_{\phi}}, n_{\phi}=n+n_{\phi_{\mathrm{p}}}+n_{y}$, one can write [6, (17), and 20, in a compact form as

$$
\dot{\phi}(t)=F_{\mathrm{r}} \phi(t)+G_{\mathrm{r}} c_{0}(t)+G e(t), \quad \phi(0)=\phi_{0}
$$

where

$$
\begin{aligned}
& F_{\mathrm{r}} \triangleq {\left[\begin{array}{ccc}
A_{\mathrm{r}} & -B_{\mathrm{r}} J_{\mathrm{c}} M_{\mathrm{p}} & B_{\mathrm{r}} H_{\mathrm{c}} \\
G_{\mathrm{p}} N & F_{\mathrm{p}} & 0 \\
0 & G_{\mathrm{c}} M_{\mathrm{p}} & F_{\mathrm{c}}
\end{array}\right] \in \mathbb{R}^{\left(n+n_{\phi_{\mathrm{p}}}+n_{y}\right) \times\left(n+n_{\phi_{\mathrm{p}}}+n_{y}\right)}, } \\
& G_{\mathrm{r}} \triangleq\left[\begin{array}{c}
B_{\mathrm{r}} J_{\mathrm{c}} \\
0 \\
-G_{\mathrm{c}}
\end{array}\right] \in \mathbb{R}^{\left(n+n_{\phi_{\mathrm{p}}}+n_{y}\right) \times n_{c 0}}, \quad G \triangleq\left[\begin{array}{c}
0 \\
G_{\mathrm{p}} N \\
0
\end{array}\right] \in \mathbb{R}^{\left(n+n_{\phi_{\mathrm{p}}}+n_{y}\right) \times n},
\end{aligned}
$$

with $N=\left[I_{n_{\mathrm{p}} \times n_{\mathrm{p}}}, 0_{n_{\mathrm{p}} \times n_{\mathrm{c}}}\right]$.

For the human loop, we consider a general class of linear human models with constant time-delay [21]

$$
\begin{aligned}
\dot{\xi}(t) & =A_{\mathrm{h}} \xi(t)+B_{\mathrm{h}} \theta(t-\tau), \quad \xi(0)=\xi_{0} \\
c_{0}(t) & =C_{\mathrm{h}} \xi(t)+D_{\mathrm{h}} \theta(t-\tau),
\end{aligned}
$$

where $\xi(t) \in \mathbb{R}^{n_{\xi}}$ is the internal human state vector, $\tau \in \mathbb{R}_{+}$is the human reaction time-delay, $A_{\mathrm{h}} \in \mathbb{R}^{n_{\xi} \times n_{\xi}}, B_{\mathrm{h}} \in \mathbb{R}^{n_{\xi} \times n_{\mathrm{r}}}$, $C_{\mathrm{h}} \in \mathbb{R}^{n_{c 0} \times n_{\xi}}$, and $D_{\mathrm{h}} \in \mathbb{R}^{n_{c 0} \times n_{\mathrm{r}}}$. Here, The input to the human dynamics is given by

$$
\theta(t)=r(t)-E_{\mathrm{h}} \phi_{\mathrm{p}}(t)
$$

where $\theta(t) \in \mathbb{R}^{n_{\mathrm{r}}}$, and $r(t) \in \mathbb{R}^{n_{\mathrm{r}}}$ is the bounded reference signal. In (28), $E_{\mathrm{h}} \in \mathbb{R}^{n_{\mathrm{r}} \times n}$ selects the appropriate states to be compared with $r(t)$.

\section{Overview of the Set-theoretic Model Reference Adaptive Control for Human-in-the-loop Systems: Stability and Performance Guarantees}

In this section, we overview the results presented in [15] for establishing the stability and guaranteeing a user-defined performance constraint on the norm of system error trajectories. Specifically, the proposed update law in (15) is predicated on the set-theoretic model reference adaptive control architecture presented in [18] in order to impose a user-defined performance bound on the system error vector. Unlike a common update law in standard model reference adaptive control, the term $\phi_{d}\left(\|e(t)\|_{P}\right)$ in [15], defined in Definition 2, serves as an error dependent learning rate. As a result, the user-defined performance guarantee at the inner loop can be shown by considering the energy function $V(e, \tilde{W})=\phi\left(\|e\|_{P}\right)+\gamma^{-1} \operatorname{tr}\left[\left(\tilde{W} \Lambda^{1 / 2}\right)^{\mathrm{T}}\left(\tilde{W} \Lambda^{1 / 2}\right)\right]$ as shown in [18]. Specifically, the time derivative of this energy function is upper bounded by $\dot{V}(e(t), \tilde{W}(t)) \leq-\frac{1}{2} \alpha_{1} V(e, \tilde{W})+\alpha_{2}$, where $\alpha_{1} \triangleq \frac{\lambda_{\min }(R)}{\lambda_{\max }(P)}, d \triangleq 2 \gamma^{-1} \tilde{w} \dot{w}\|\Lambda\|_{2}$, $\alpha_{2} \triangleq \frac{1}{2} \alpha_{1} \gamma^{-1} \tilde{w}^{2}\|\Lambda\|_{2}+d$, and $\tilde{w}=\hat{W}_{\max }+w$. Hence, $V(e, \tilde{W})$ is upper bounded and if $\left\|e_{0}\right\|_{P}<\epsilon$, then the system error satisfies the strict user-defined bound given by

$$
\|e(t)\|_{P}<\epsilon, \quad t \geq 0 .
$$

In order to establish the overall stability of the proposed control architecture, we let $x_{0}(t) \triangleq\left[\phi^{\mathrm{T}}(t), \xi^{\mathrm{T}}(t)\right]^{\mathrm{T}} \in \mathbb{R}^{n_{0}}$, $n_{0} \triangleq n_{\phi}+n_{\xi}$, and we write the dynamics in 23) and 26 as

$$
\dot{x}_{0}(t)=A_{0} x_{0}(t)+A_{1} x_{0}(t-\tau)+B_{0} r(t-\tau)+B_{1} e(t), \quad x_{0}(t)=\psi_{0}(t) \text { for } t \in[-\tau, 0],
$$

where $\psi_{0}(t) \in \mathbb{R}^{n_{0}}$ is the initial condition and

$$
\begin{aligned}
& A_{0} \triangleq\left[\begin{array}{cc}
F_{\mathrm{r}} & G_{\mathrm{r}} C_{\mathrm{h}} \\
0 & A_{\mathrm{h}}
\end{array}\right] \in \mathbb{R}^{n_{0} \times n_{0}}, \quad A_{1} \triangleq\left[\begin{array}{cc}
-G_{\mathrm{r}} D_{\mathrm{h}} E_{\mathrm{h}} N_{0} & 0 \\
-B_{\mathrm{h}} E_{\mathrm{h}} N_{0} & 0
\end{array}\right] \in \mathbb{R}^{n_{0} \times n_{0}}, \\
& B_{0} \triangleq\left[\begin{array}{c}
G_{\mathrm{r}} D_{\mathrm{h}} \\
B_{\mathrm{h}}
\end{array}\right] \in \mathbb{R}^{n_{0} \times n_{\mathrm{r}}} \quad, \quad B_{1} \triangleq\left[\begin{array}{c}
G \\
0
\end{array}\right] \in \mathbb{R}^{n_{0} \times n},
\end{aligned}
$$


with $N_{0}=\left[0_{n_{\phi_{\mathrm{p}}} \times n}, I_{n_{\phi_{\mathrm{p}}} \times n_{\phi_{\mathrm{p}}}}, 0_{n_{\phi_{\mathrm{p}}} \times n_{y}}\right]$. As standard, the overall nominal system performance corresponds to the case when there is no uncertainty in the system and consequently, the error signal vanishes. Hence, one can consider the ideal behavior dynamics for 30 given by

$$
\dot{\hat{x}}_{0}(t)=A_{0} \hat{x}_{0}(t)+A_{1} \hat{x}_{0}(t-\tau)+B_{0} r(t-\tau), \quad \hat{x}_{0}(t)=\hat{\psi}_{0}(t) \text { for } t \in[-\tau, 0] .
$$

Now letting $\tilde{x}(t) \triangleq x_{0}(t)-\hat{x}_{0}(t)$ and using $(30)$ and $(33)$, the error dynamics can be written as

$$
\dot{\tilde{x}}(t)=A_{0} \tilde{x}(t)+A_{1} \tilde{x}(t-\tau)+B_{1} e(t), \quad \tilde{x}(t)=\psi(t) \text { for } t \in[-\tau, 0],
$$

where $\psi(t) \triangleq \psi_{0}(t)-\hat{\psi}_{0}(t)$. Setting $e(t)=0$ in [34, the nominal system is given by

$$
\dot{\tilde{x}}(t)=A_{0} \tilde{x}(t)+A_{1} \tilde{x}(t-\tau) .
$$

Without loss of generality, we assume that this nominal system is asymptotically stablef Let $\Psi(t) \in \mathbb{R}^{n_{0} \times n_{0}}$ to be the fundamental solution of the nominal system in (35) satisfying

$$
\dot{\Psi}(t)=A_{0} \Psi(t)+A_{1} \Psi(t-\tau)
$$

with the initial condition $\Psi(0)=I$ and $\Psi(t)=0$ for $t<0$. Furthermore, it follows from the asymptotically stability of the nominal system in (35) that there exist an $\alpha>0$ such that $\|\Psi(t)\|_{2} \leq K e^{-\alpha t}$ for some $K>1$. We can now apply Comment 4 of [15] to bound the error dynamics in (34) given by

$$
\|\tilde{x}(t, \psi)\|_{2} \leq K\|\psi(0)\|_{2}+\frac{K}{\alpha}\left[\left\|A_{1}\right\|_{2} \bar{\psi}\left(e^{\alpha \tau}-1\right)+\left\|B_{1}\right\|_{2} \frac{\epsilon}{\sqrt{\lambda_{\min }(P)}}\right]
$$

where $\bar{\psi} \triangleq \sup _{-\tau \leq \theta \leq 0} \psi(\theta)$. Assuming that the initial condition of the system in 30 is equal to that of the ideal behavior of the system in (33) (i.e. $\bar{\psi}=0$ ), one can further simplify (37) as

$$
\|\tilde{x}(t, \psi)\|_{2} \leq \epsilon \mu, \quad \mu \triangleq \frac{K\left\|B_{1}\right\|_{2}}{\alpha \sqrt{\lambda_{\min }(P)}} .
$$

The upper bound on the error signal $\tilde{x}(t, \psi)$ obtained in (38) implies that the user-defined performance parameter $\epsilon$ can be utilized to control the deviation of the system from the ideal behavior in (33).

\section{Pilot Experimental Results}

The objective in this section is to investigate whether or not the above presented theoretical approach has promise. To this end, before conducting a comprehensive experimental study, five volunteers from the authors' research team tested the designed computer-based game, at a preliminary level. The following thus represents only a piloting of this effort, and should not be perceived to be a general experimental study. Since the volunteers are from the authors' research team, it was not possible to avoid bias although we do believe that the volunteers fairly played the simulation study. The following results should be considered in view of these remarks.

What we wish to find out is whether or not the human response deviates less from a nominal one as the system error bound $\epsilon$ decreases. To this end, volunteers tested a series of games on a computer-simulated dynamic system via a mouse interface. The computer-simulated dynamics is the linearized longitudinal flight dynamics for which the details can be found in [15]. The system output is the pitch angle $\theta(t)$ of the vehicle. The volunteers are supposed to drive the pitch angle to a predetermined final value which was initially zero. The mouse interface helps volunteers to generate a pitch rate command $c_{o}(t)=\theta_{\mathrm{cmd}}(t)$. Current pitch rate $\theta(t)$ and the desired reference pitch rate $r(t)$ was displayed on the computer screen to provide visual feedback.

Screenshot of the computer program can be seen in Figure 2. The details of the program are summarized as follows:

- Current pitch rate $\theta(t)$ is the output of the system and visualized with a red box (\#1).

- Reference value $r(t)$ equals to $15^{\circ}$ and it is visualized with a black box (\#2).

- Pitch command $\theta_{\mathrm{cmd}}(t)=c_{o}(t)$ which is the human output, and it is visualized with a red bar (\#3). Experiments are taken in two sequential phases:

\footnotetext{
*We refer to Comment 3 of [15] for stability analysis of the nominal system dynamics in 35] and discussions on finding critical delay values.
} 


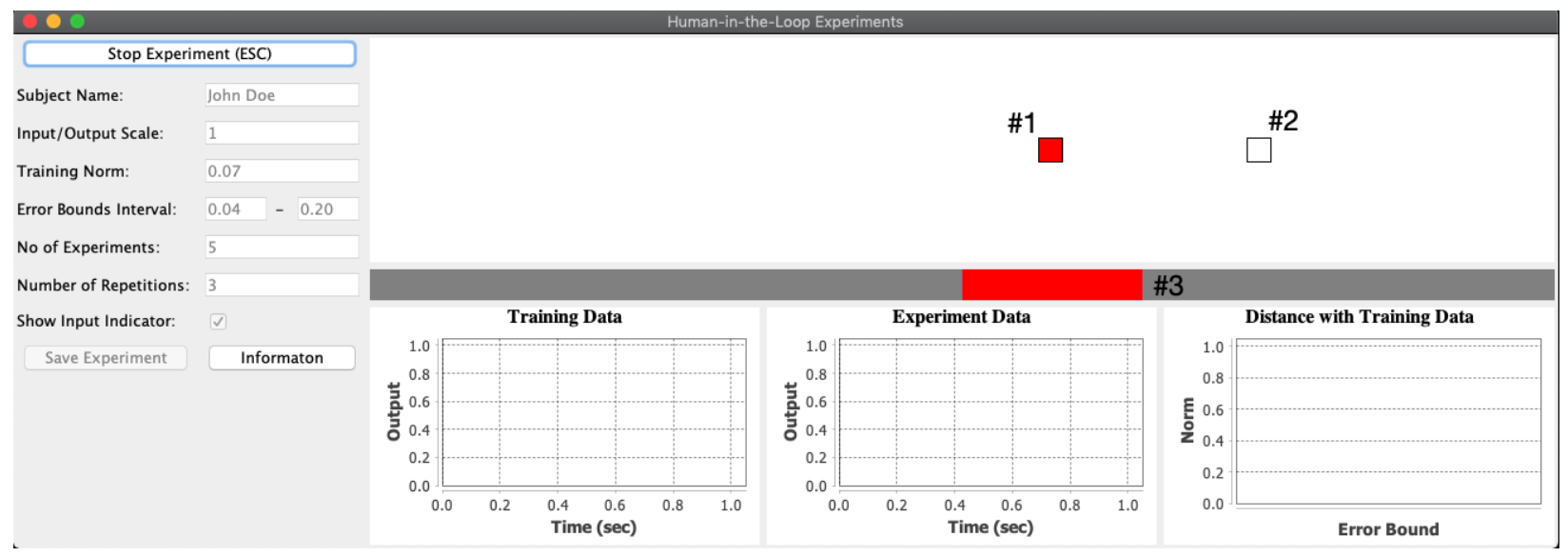

Fig. 2 Simulation screen. Notice that the three plots are populated only after all the 15 trials are completed, and hence the volunteers do not receive feedback on their performance after each trial.

- Training Phase (Phase I): In this phase, the subjects are trained with the reference model. There is no uncertainty, and the dynamic model is an LTI system. For the $i^{\text {th }}$ trial in the training phase, pitch rate $\theta_{i}^{\text {tr }}(t)$, pitch command $\theta_{\mathrm{cmd}, i}^{\mathrm{tr}}(t)$, and the time $t_{i}^{\mathrm{tr}}$ are recorded. Let index of last three trials be 1,2, and 3. Training phase is concluded when

$$
\max _{i, j \in\{1,2,3\}}\left\|\theta_{i}^{\mathrm{tr}}(t)-\theta_{j}^{\mathrm{tr}}(t)\right\|_{\infty}<\text { training_norm }=0.07
$$

for $i, j \in\{1,2,3\}$. Then, the nominal system output $\theta^{\text {nom }}(t)=\frac{1}{3} \sum_{i=1}^{3} \theta_{i}^{\operatorname{tr}}(t)$ and the nominal human output $\theta_{\mathrm{cmd}}^{\mathrm{nom}}(t)=\frac{1}{3} \sum_{i=1}^{3} \theta_{\mathrm{cmd}, i}^{\mathrm{tr}}(t)$ represent the nominal behavior of the pilot.

- Test Phase (Phase II): In this phase, the system is uncertain. The proposed set-theoretic model reference adaptive control is active and is put to test. Volunteers were given the same task as in the training phase, and have performed the task for 5 different system error bounds $(\epsilon \in\{0.04,0.08,0.12,0.16,0.20\})$, each repeated three times but all in random order. For the $i^{t h}$ trial with an error bound of $\epsilon$, the pitch rate $\theta_{\epsilon, i}^{\exp }(t)$, pitch command $\theta_{\mathrm{cmd}, \epsilon, i}^{\exp }(t)$, and the time $t_{\epsilon, i}^{\exp }$ are recorded. At the end of this phase, the best trial for each $\epsilon$ is determined as follows

$$
\theta_{\epsilon}^{\exp }(t)=\theta_{\epsilon, i}^{\exp }(t), \text { such that }\left\|\theta_{\epsilon, i}^{\exp }(t)-\theta^{\text {nom }}(t)\right\|_{\infty} \leq\left\|\theta_{\epsilon, j}^{\exp }(t)-\theta^{\text {nom }}(t)\right\|_{\infty}, \quad i, j \in\{1,2,3\} .
$$

In other words, we select the waveform that is the closest to the nominal waverform in the sense of infinity norm. The reason for this selection is explained as follows. Notice that in the training phase, the dynamics is free of nonlinearities and hence the human behavior is considered to be "nominal." What we wish to find out is how well the humans can maintain their nominal behavior despite the nonlinearities introduced in the test phase and how well we can potentially modulate this similarity through set-theoretic adaptive control. This selection criterion also allows one to eliminate the waveforms from trials in which the subjects performed poorly and/or could not start the experiments on time as desired.

In Figure 3, the training-phase data $\theta_{i}^{\mathrm{tr}}(t), \theta^{\mathrm{nom}}(t)$ and the test-phase data $\theta_{\epsilon}^{\exp }(t)$ are plotted. The distance with nominal response for each subject with respect to different system error bounds can be seen in Figure 5. This figure shows that the output of the nonlinear dynamics deviates much less from the nominal dynamics as we tighten the error bound by decreasing $\epsilon$ in the set-theoretic adaptive control. These results indicate that indeed it is possible to bring the behavior of the nonlinear dynamics closer to a nominal one through this control approach.

Finally, we investigate whether or not similar qualitative arguments can be made in terms of human behavior. To this end, we present Fig 4 where we plot $\theta_{\mathrm{cmd}, i}^{\mathrm{tr}}(t), \theta_{\mathrm{cmd}}^{\text {nom }}(t)$, and $\theta_{\mathrm{cmd}, \epsilon, i}^{\exp }(t)$. The figure shows that it is not possible to argue that human output $\theta_{\mathrm{cmd}}$ deviates from the trained (nominal) behavior despite the added uncertainties in the test phase. This observation differs from what was observed from the output of the nonlinear dynamics. Moreover, since the human behavior does not seem to substantially change despite those uncertainties (see Fig 6), the general outcome of the behavior in the nonlinear dynamics becomes poorer with increased uncertainties (that is, the human does not seem to carefully correct the dynamics in the presence of uncertainties). These results also demonstrate that set-theoretic model reference adaptive control is instrumental in keeping the nonlinear dynamics closer to the nominal one, so that 
the human decisions, which are biased toward the nominal model, can still yield better performance from the nonlinear dynamics; this is clearly observed in cases when the tolerance is tightened by selecting smaller $\epsilon$ values.

Volunteer 1 Training Phase

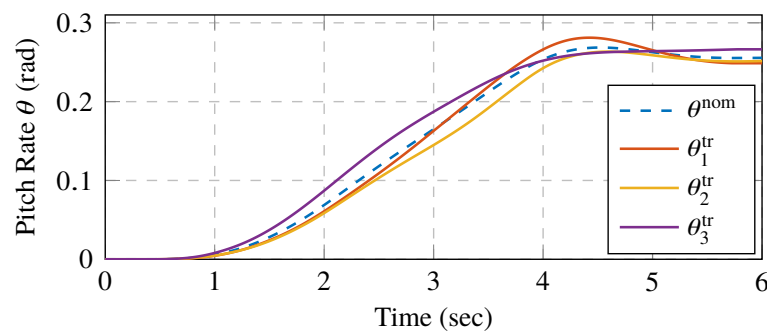

Volunteer 2 Training Phase

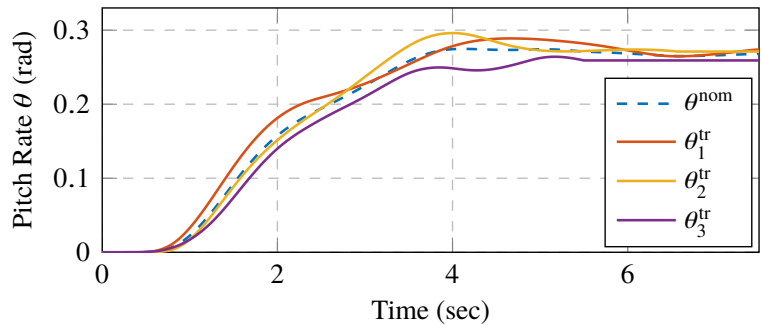

Volunteer 3 Training Phase

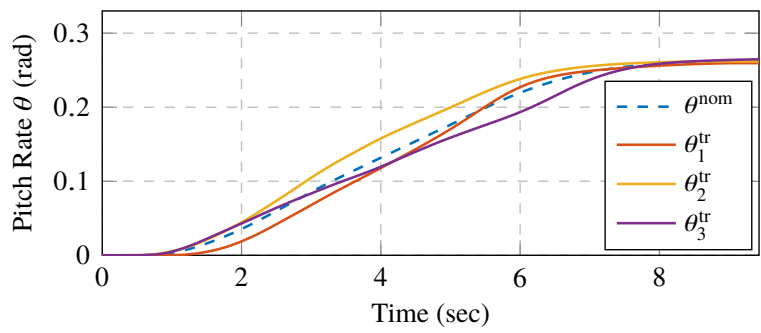

Volunteer 4 Training Phase

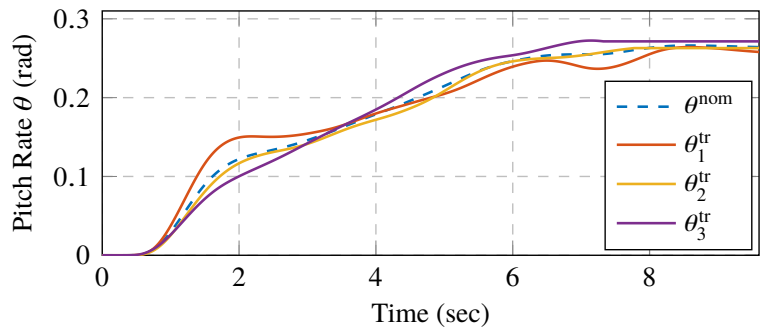

Volunteer 5 Training Phase

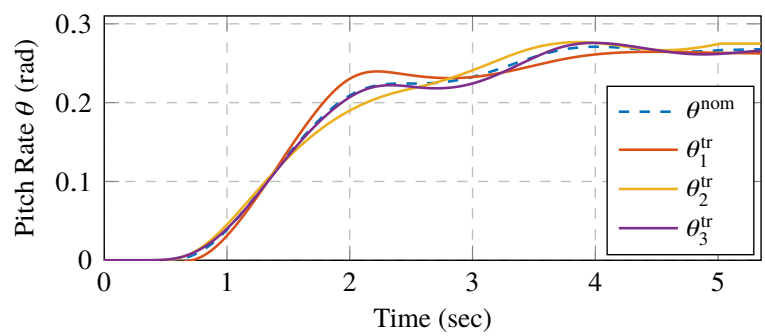

Volunteer 1 Test Phase

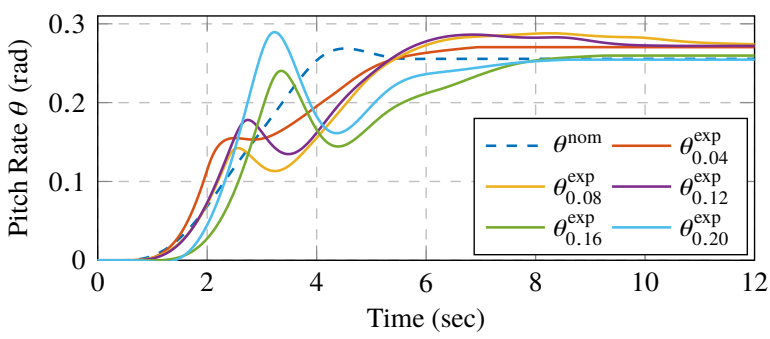

Volunteer 2 Test Phase

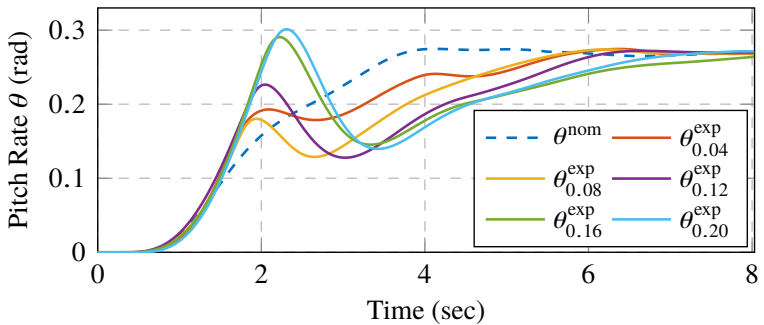

Volunteer 3 Test Phase

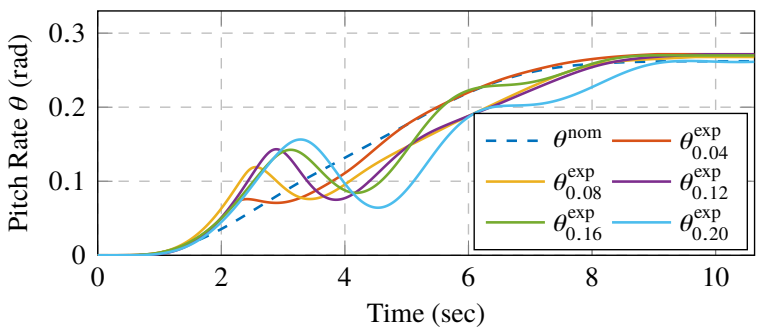

Volunteer 4 Test Phase

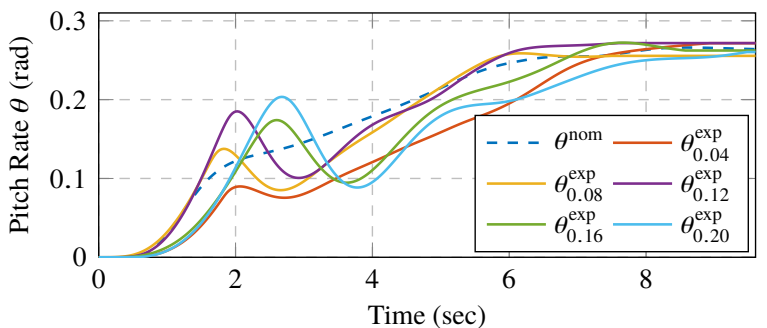

Volunteer 5 Test Phase

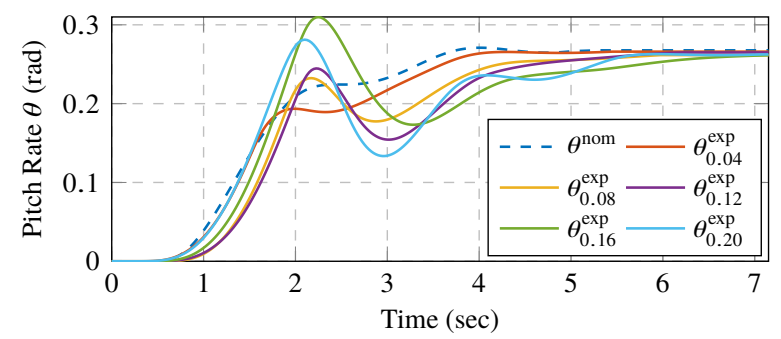

Fig. 3 The system outputs $\theta(t)$ recorded in training and test phases for each volunteer. 
Volunteer 1 Training Phase

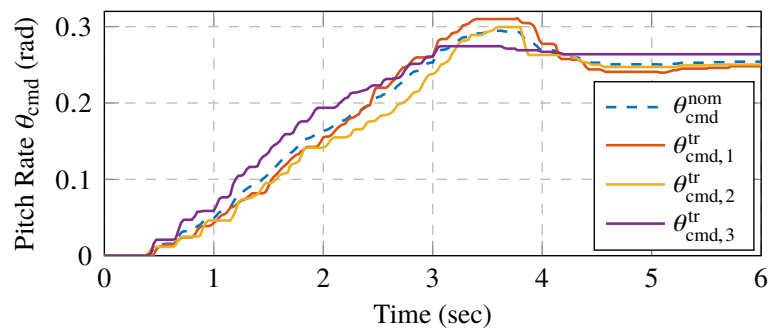

Volunteer 2 Training Phase

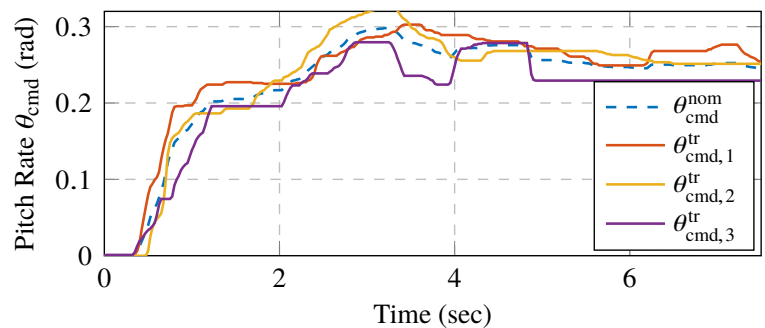

Volunteer 3 Training Phase

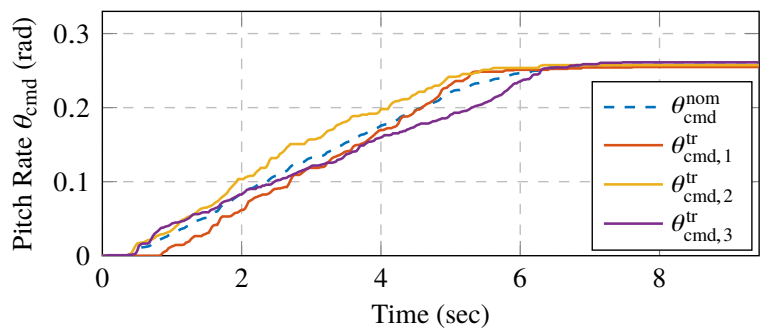

Volunteer 4 Training Phase

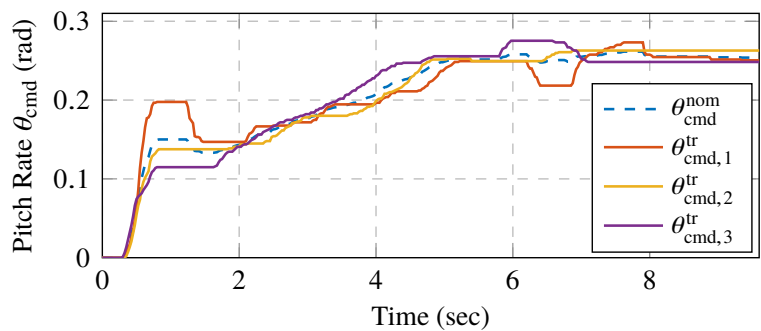

Volunteer 5 Training Phase

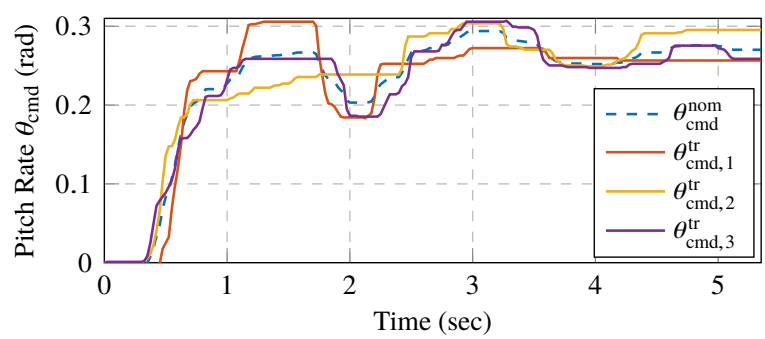

Volunteer 1 Test Phase

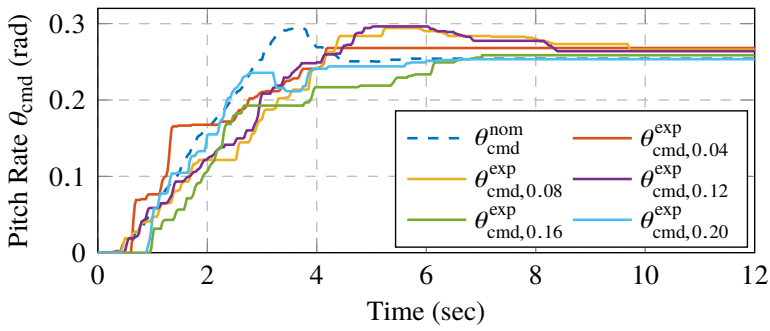

Volunteer 2 Test Phase

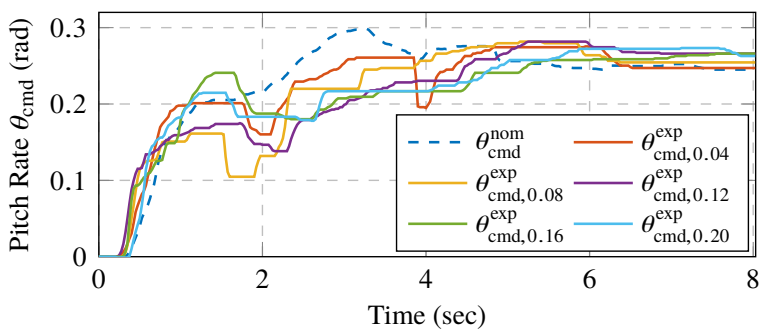

Volunteer 3 Test Phase

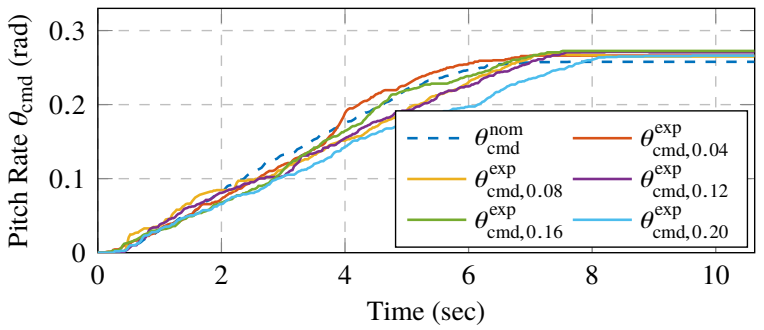

Volunteer 4 Test Phase

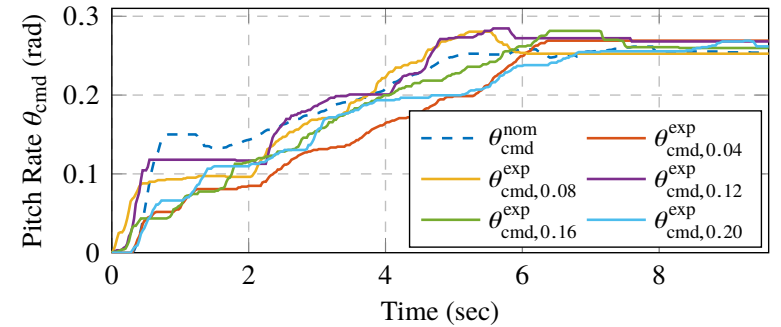

Volunteer 5 Test Phase

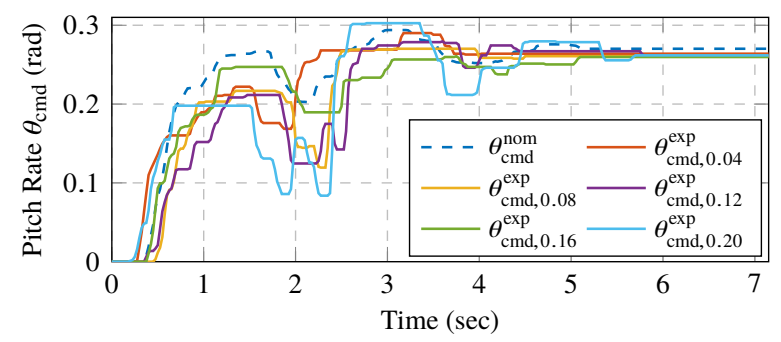

Fig. 4 The human outputs $\theta_{\mathrm{cmd}}(t)$ recorded in training and test phases for each volunteer. 

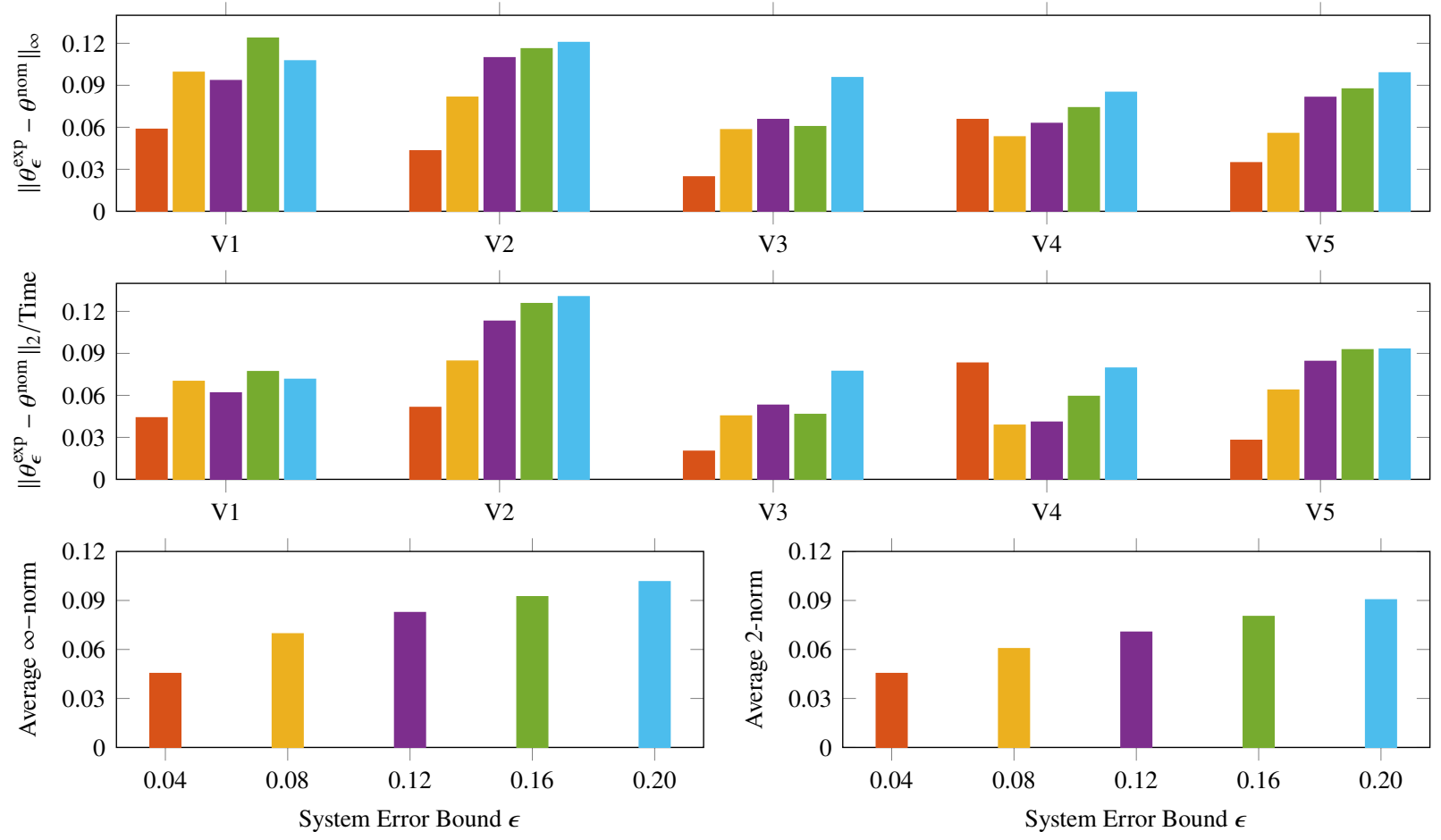

Fig. 5 Distance between system output and nominal output in terms of infinity and 2 norms with respect to system error upper bound $\epsilon$. It can be seen that, the system output deviates less as $\epsilon$ gets lower.
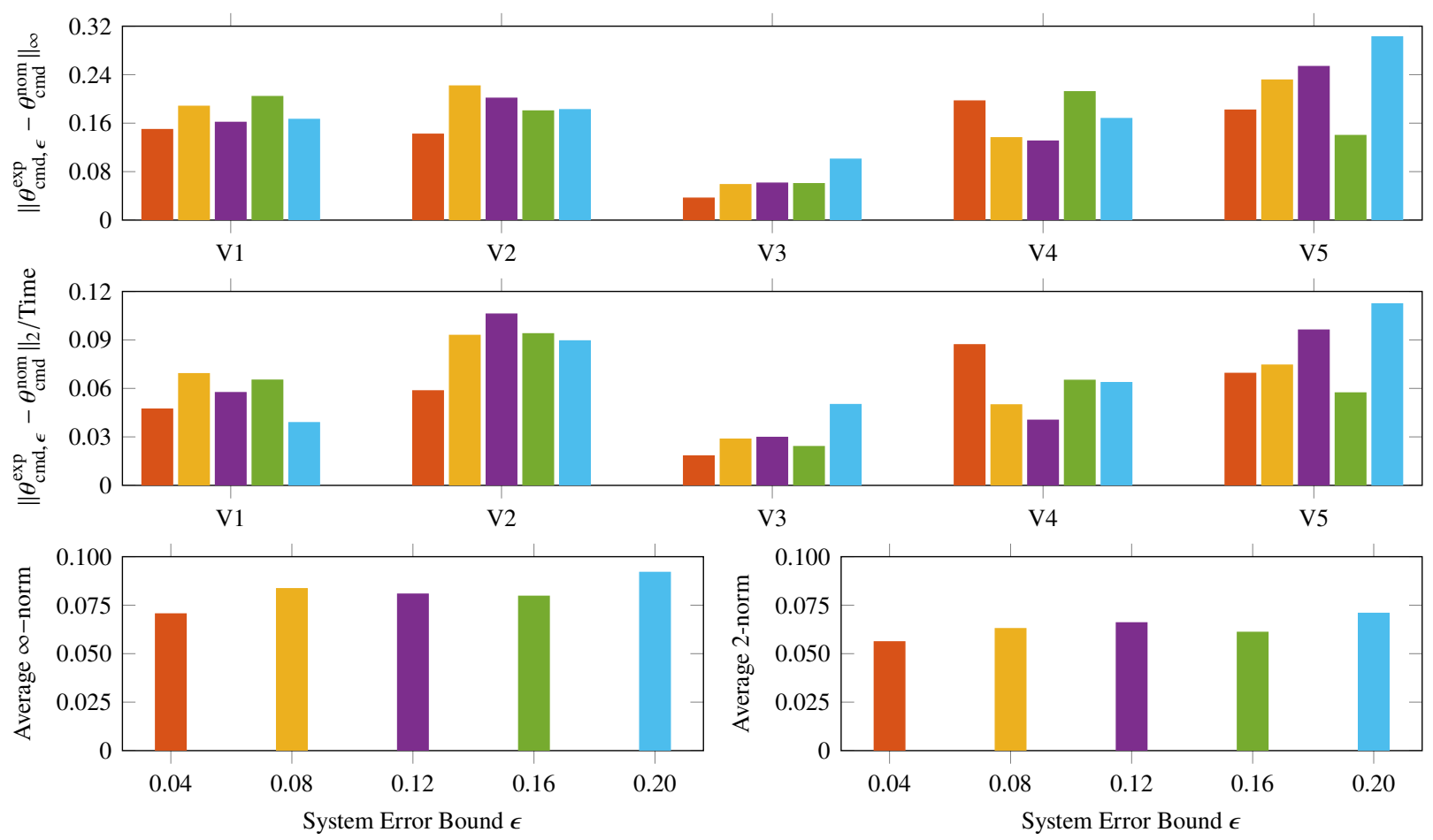

Fig. 6 Distance between human output and nominal human output in terms of infinity and 2-norms with respect to system error upper bound $\epsilon$. 


\section{Conclusion}

Authors' prior work on set-theoretic adaptive control prescribes a control with which one can tighten a tolerance metric in order to drive a nonlinear system as close as possible to a nominal system. This approach has the potential to advance the synergy between humans and machines and is put to test on a limited, preliminary pilot study in which volunteers of the research team tested a Java based game. The arising data in this testing provides evidence that indeed by tightening the metric in the set-theoretic adaptive control framework, the behavior of the nonlinear dynamics can be made closer to their respective nominal behavior. While we acknowledge that the presented data is from the research team, could be inadvertently biased, and is far from generalization, it indicates that it might be possible to adapt the nonlinear dynamics to a nominal one, simplifying thus the workload of humans when interacting with such nonlinear dynamics. This result therefore suggests that one can in the future train human subjects only on nominal dynamic systems but utilize a set-theoretic adaptive controller to assist humans in better performing tasks that require controlling dynamics with nonlinearities. Future work will focus on a comprehensive study with human subjects experiments to statistically quantify the advantages of the proposed controller for human subjects, and to prescribe effective training modules for human subjects. This perspective has to potential to yield better closed-loop performance in human-in-the-loop systems, shorter training periods for human subjects, and faster transition of subjects from training to real-life operations.

\section{Acknowledgments}

This research was supported by the Dynamics, Control, and Systems Diagnostics Program of the National Science Foundation under Grant CMMI-1657637.

\section{References}

[1] Li, W., Sadigh, D., Sastry, S. S., and Seshia, S. A., "Synthesis for human-in-the-loop control systems," International Conference on Tools and Algorithms for the Construction and Analysis of Systems, Springer, 2014, pp. 470-484.

[2] Koru, A. T., Yucelen, T., Sipahi, R., Ramírez, A., and Dogan, K. M., "Stability of Human-in-the-Loop Multiagent Systems with Time Delays," In Proceedings of American Control Conference (ACC), 2019, pp. 4854-4859.

[3] McRuer, D. T., and Jex, H. R., "A review of quasi-linear pilot models," IEEE transactions on human factors in electronics, Vol. HFE-8, No. 3, 1967, pp. 231-249.

[4] Kleinman, D., Baron, S., and Levison, W., "An optimal control model of human response part I: Theory and validation," Automatica, Vol. 6, No. 3, 1970, pp. 357-369.

[5] Schmidt, D., and Bacon, B., "An optimal control approach to pilot/vehicle analysis and the Neal-Smith criteria," Journal of Guidance Control Dynamics, Vol. 6, 1983, pp. 339-347.

[6] Hess, R., and Modjtahedzadeh, A., "A control theoretic model of driver steering behavior," IEEE Control Systems Magazine, Vol. 10, No. 5, 1990, pp. 3-8.

[7] Hess, R. A., "Unified theory for aircraft handling qualities and adverse aircraft-pilot coupling," Journal of Guidance, Control, and Dynamics, Vol. 20, No. 6, 1997, pp. 1141-1148.

[8] Thurling, A. J., "Improving UAV handling qualities using time delay compensation," Tech. rep., Air Force Inst of Tech Wright-Patterson AFB, 2000.

[9] Witte, J. B., "An investigation relating longitudinal pilot-induced oscillation tendency rating to describing function predictions for rate-limited actuators," Tech. rep., Air Force Inst of Tech Wright-Patterson AFB, 2004.

[10] Munir, S., Stankovic, J. A., Liang, C.-J. M., and Lin, S., "Cyber physical system challenges for human-in-the-loop control," Presented as part of the 8th International Workshop on Feedback Computing, San Jose, CA, USA, 2013.

[11] Suzuki, S., Kurihara, K., Furuta, K., Harashima, F., and Pan, Y., "Variable dynamic assist control on haptic system for human adaptive mechatronics," Proceedings of the 44th IEEE Conference on Decision and Control, IEEE, 2005, pp. 4596-4601.

[12] Ikemoto, S., Amor, H. B., Minato, T., Jung, B., and Ishiguro, H., "Physical human-robot interaction: Mutual learning and adaptation," IEEE robotics \& automation magazine, Vol. 19, No. 4, 2012, pp. 24-35.

[13] Adloo, H., Noroozi, N., and Karimaghaee, P., "Observer-based model reference adaptive control for unknown time-delay chaotic systems with input nonlinearity,” Nonlinear dynamics, Vol. 67, No. 2, 2012, pp. 1337-1356. 
[14] Arabi, E., and Yucelen, T., "Set-Theoretic Model Reference Adaptive Control with Time-Varying Performance Bounds," International Journal of Control, Vol. 92, No. 11, 2019, pp. 2509-2520.

[15] Arabi, E., Yucelen, T., Sipahi, R., and Yildiz, Y., "Human-in-the-Loop Systems with Inner and Outer Feedback Control Loops: Adaptation, Stability Conditions, and Performance Constraints," AIAA Guidance, Navigation, and Control Conference, San Diego, CA, USA, 2019.

[16] Lavretsky, E., and Wise, K., Robust and adaptive control with aerospace applications, Springer Science \& Business Media, 2012.

[17] Pomet, J.-B., and Praly, L., "Adaptive nonlinear regulation: Estimation from the Lyapunov equation," IEEE Transactions on Automatic Control, Vol. 37, No. 6, 1992, pp. 729-740.

[18] Arabi, E., Gruenwald, B. C., Yucelen, T., and Nguyen, N. T., "A set-theoretic model reference adaptive control architecture for disturbance rejection and uncertainty suppression with strict performance guarantees," International Journal of Control, Vol. 91, No. 5, 2018, pp. 1195-1208.

[19] Wiese, D. P., “Systematic adaptive control design using sequential loop closure," Ph.D. thesis, Massachusetts Institute of Technology, 2016.

[20] Wiese, D. P., Annaswamy, A. M., Muse, J. A., Bolender, M. A., and Lavretsky, E., "Sequential loop closure based adaptive autopilot design for a hypersonic vehicle," AIAA Guidance, Navigation, and Control Conference, San Diego, CA, USA, 2016.

[21] Yucelen, T., Yildiz, Y., Sipahi, R., Yousefi, E., and Nguyen, N., "Stability limit of human-in-the-loop model reference adaptive control architectures," International Journal of Control, Vol. 91, No. 10, 2018, pp. 2314-2331. 